\title{
The Galactic cosmic-ray Sun shadow observed by HAWC
}

\author{
Olivia Enríquez ${ }^{a}$ and Alejandro Lara ${ }^{a *}$ for the HAWC collaboration ${ }^{b}$ \\ ${ }^{a}$ Instituto de Geofísica, Universidad Nacional Autónoma de México \\ ${ }^{b}$ For a complete author list, see www.hawc-observatory.org/collaboration/icrc2015.php. \\ E-mail: olienriquezrivera@gmail.comalara.unamegmail.com
}

\begin{abstract}
The magnetic field of the Solar corona is difficult to measure directly. However, indirect observations of the solar corona are possible using the deficit in flux of cosmic rays coming from the direction of the Sun. Low-energy cosmic rays $(\sim \mathrm{GeV})$ are deflected by the inner magnetic field of the Sun and the interplanetary magnetic field frozen into the solar wind. In contrast, high-energy cosmic rays ( $\sim \mathrm{TeV}$ and above) are absorbed in the Sun's photosphere producing a shadow in the Sun's nominal position viewed from Earth. Several ground-based instruments have observed the effects of the heliospheric magnetic field on the size of the sun shadow and its position. The High-Altitude Water Cherenkov Observatory (HAWC) is an air shower array located in the central region of Mexico that observes $\mathrm{TeV}$ cosmic rays at a rate of about $15 \mathrm{kHz}$. In this work, we present preliminary images of the sun shadow from data collected by HAWC during 2013 and 2014 for different energy ranges.
\end{abstract}

The 34th International Cosmic Ray Conference,

30 July- 6 August, 2015

The Hague, The Netherlands

\footnotetext{
* Speaker.
} 


\section{Introduction}

For an observer on Earth, the Sun and the Moon block a portion of the Galactic cosmic ray (GCR) flux casting a shadow equal to their physical angular size [1], both roughly $0.5^{\circ}$ in diameter. The Moon shadow has been used to calibrate the pointing accuracy and angular resolution of air shower detectors as early as 1991 [2, 3]. An absence of a magnetic field around the Moon allows cosmic rays to travel from the Moon to the Earth without large deflections. The Sun has a variable magnetic field which can strongly scatter nearby charged cosmic rays affecting the geometrical form of the observed Sun shadow. The time dependence of this field, the so-called "11-year Solar cycle," changes the shape and intensity of the observed Sun shadow. This allows for the study of the temporal behavior of the Solar magnetic field.

The Sun shadow was observed by the Tibet array [4, 5, 6, 7] and the ARGO Experiment [8]. The authors performed comprehensive studies with data throughout the 11-year Solar cycle and, using simulations, they showed that it is possible to obtain information about the Solar magnetic field with the GCR Sun shadow [7]. The Milagro Experiment also reported the observation of the Sun shadow $[9,10]$.

The newly finished High-Altitude Water Cherenkov (HAWC) Observatory is able to report on the angular observations of $100 \mathrm{GeV}$ to $100 \mathrm{TeV}$ cosmic rays. At these energies, cosmic rays are confined within the Galaxy and are believe to be of Galactic origin. Using the first 30 detectors of HAWC, observation of the Moon shadow was in good agreement with simulations [11]. In this work, we present the observations of the Sun shadow with a larger number of detectors (between 95 and 111), which allow us to make preliminary estimations of GCR energy.

In Section 2, we describe HAWC as a GCR detector. Section 3 details the method for producing the Sun shadow maps from data. HAWC Sun shadow maps, using data acquired during construction, are presented in Section 4. Finally, we conclude and describe future plans for measuring the coronal magnetic field using the analysis of the Sun shadow in Section 5.

\section{HAWC}

The HAWC Observatory is located at $4100 \mathrm{~m}$ above the sea level on the Sierra Negra Volcano in the central part of Mexico (N $18^{\circ} 59^{\prime} 48^{\prime \prime}$, W $97^{\circ} 18^{\prime} 34^{\prime \prime}$ ). HAWC consists of 300 water Cherenkov detectors (WCD) covering an area of $22000 \mathrm{~m}^{2}$. Each WCD is a cylindrical container $4.5 \mathrm{~m}$ high and $7.3 \mathrm{~m}$ in diameter filled with $\sim 200000$ liters of filtered water. They are instrumented with one central high quantum efficiency 10" photo-multipliers (PMT) surrounded by three lateral 8" PMTs. The PMTs face upwards to detect Cherenkov light from relativistic particles, produced as secondaries in extensive air showers. A detailed description of HAWC is presented in Reference [12].

The HAWC detector measures the primary particle direction through accurate temporal and spatial determination of the atmospheric shower front of secondary particles. The vast majority of primary cosmic particles are cosmic rays (>99\%), therefore HAWC is a sensitive GCR detector in an energy range going from $100 \mathrm{GeV}$ to $100 \mathrm{TeV}$. HAWC can be used to instantaneously monitor more than 2 steradians (sr) of the overhead sky with a duty cycle higher than $95 \%$. This property makes it possible to continuously observe the Sun. 
The modular construction of HAWC allowed for data acquisition at different stages of construction of the array. In this work, we present the data obtained with HAWC-95 and HAWC-111, $i$. e. with 95 and 111 WCDs. We use the number of PMTs hit (nHit) by an air shower as a proxy of the energy of the primary particle. We present results of three energy-proxy bins: nHit $>30,72$ and 109. From simulation we determine the primary median energies to be $\sim 2.0,8.0$ and $50 \mathrm{TeV}$, respectively. The following discussion corresponds to these energy-proxy bins.

The Sun blocks the GCR flux also, but as it has a strong and variable magnetic field, the produced shadow is less defined. It also changes with time depending on the Solar activity. We are exploring the possibility of studying the Solar magnetic field through the Solar deficit of GCR observed by HAWC.

\section{Method}

After the first stage of data reduction, the reconstructed arrival direction of the air showers are stored in data maps that use a HEALpix binning [13] of approximately $0.1^{\circ} \times 0.1^{\circ}$ bins Suncentered coordinate system based on equatorial coordinates. Each reconstructed cosmic-ray air shower direction is given an azimuthal coordinate of its incident right ascension minus the current Sun right ascension and a polar coordinate of its incident declination minus the current Sun declination. These are referred as $\Delta \alpha$ and $\Delta \delta$.

To obtain the maps of deficits and excesses of air shower events in the sky, the data map is compared with a reference map or background map. The background map is an estimate of the response of HAWC to an isotropic cosmic ray flux. It is important to note that the background map is not itself isotropic due to atmospheric effects and the asymmetric shape of the detector [14]. Background maps are produced from the data themselves using the method of Direct Integration [15]

After the background map is created, we apply a smoothing function, a top hat function of $2^{\circ}$ radius, in order to draw out correlations in neighboring bins. Intensity maps are calculated by subtracting the background map to the data map. Each bin from these maps can be divided by the background counts to obtain relative intensity maps. Finally, we use the $\mathrm{Li}$ and Ma method [16] to find the significance of the relative intensity maps.

\section{Sun Shadow Maps}

The significance (relative intensity) maps for the different energy-proxy bins are shown in the right (left) panels in Figures 1, 2 and 3. We present Sun shadow maps from June 13, 2013 to July 09, 2013, which corresponds to HAWC-95/111. The total duration of the maps is $8121 \mathrm{hrs}$.

The size and position of the Sun shadow were obtained by fitting a 2-D asymmetric Gaussian to the relative intensity maps (Figures 1, 2 and 3). Table 1 summarizes the results of the fits.

From Figures 1, 2 and 3, it is clear that the Solar shadow has two components, defined by the energy of the GCR and the strength of the Solar magnetic field. At low GCR energies the diffusive effect of the Solar magnetic field is strong, causing a less defined shadow (Figure 1). Whereas at high energies, the GCR flux is blocked by the Solar material (Figure 3), and the Sun shadow size 

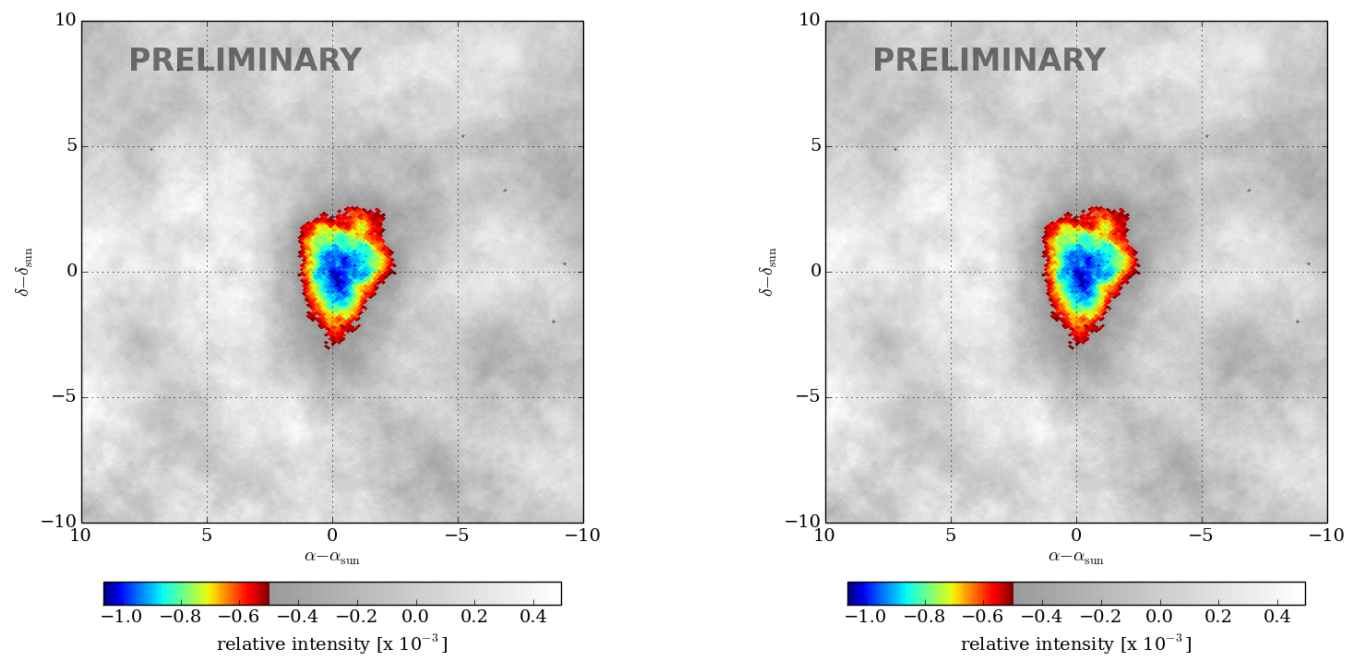

Figure 1: Relative intensity (left) and significance (right) maps of the Sun shadow with energy-proxy bins $>30 \mathrm{nHit}$ (see text for details)
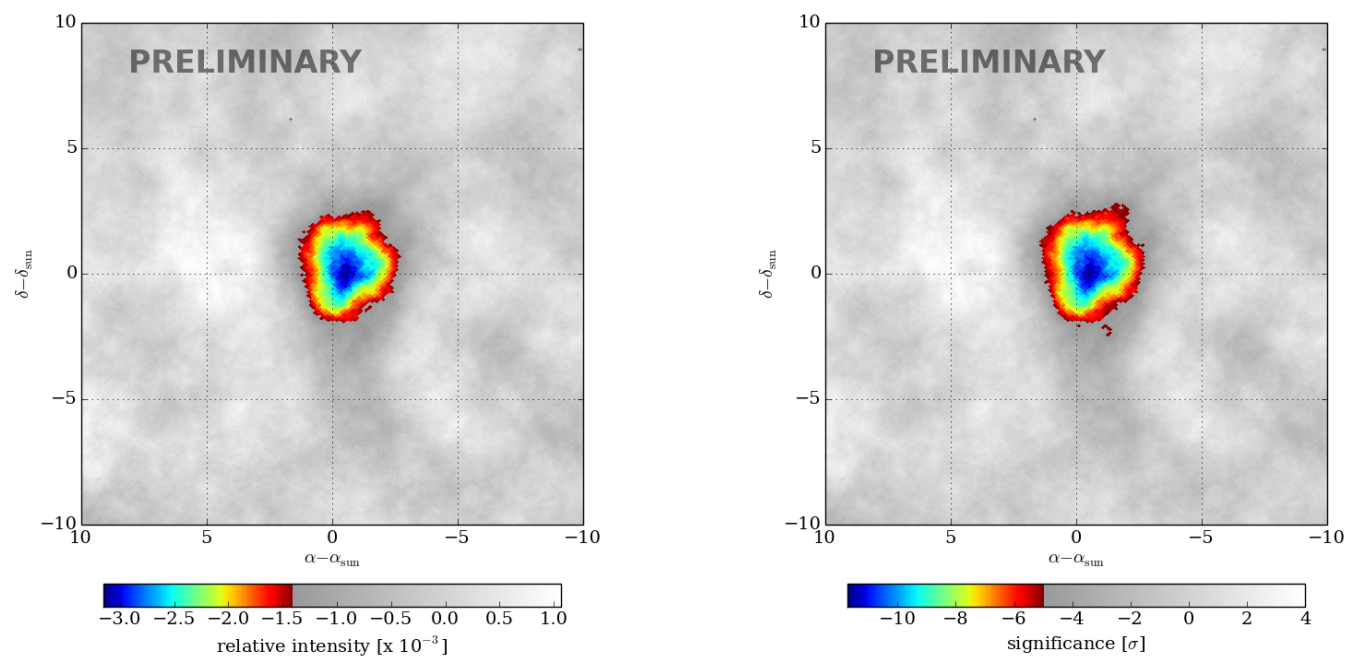

Figure 2: Relative intensity (left) and significance (right) maps of the Sun shadow with energy-proxy bins $>72 \mathrm{nHit}($ see text for details)

should approximate to that of the Moon. This is also observed in Table 1 where the widths in $\Delta \alpha$ and $\Delta \delta$ decrease, i.e. the shadow becomes better defined, as the energy-proxy bin increases.

In order to quantify the depth of the Sun shadow as the minimum energy-proxy bin changes we have plotted the differential relative intensity as a function of angular distance from the expected Sun shadow position in Figures 4 and 5. In this case a radial Gaussian fit was applied to the data. The amplitude of the fits in the plots indicate that as nHit increases the relative intensity reaches higher values. This reflects the fact that high energy GCRs contribute to a greater extent to the Sun shadow than low energy GCRs. 

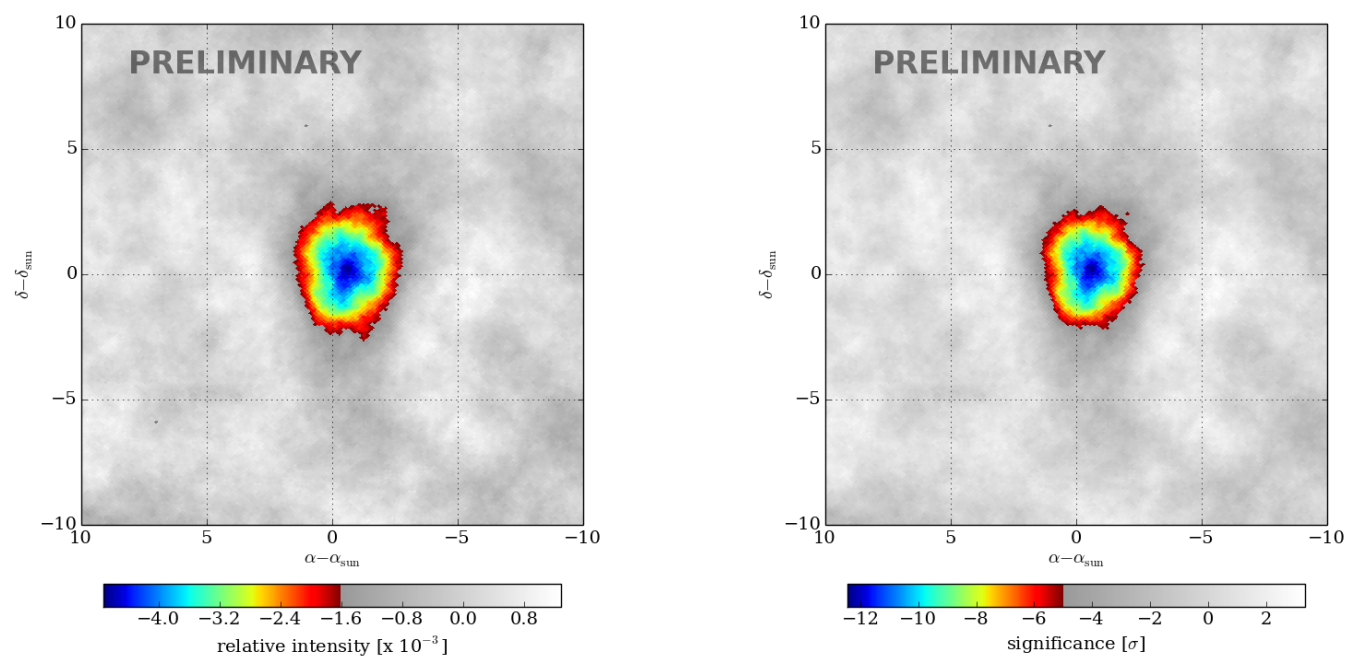

Figure 3: Relative intensity (left) and significance (right) maps of the Sun shadow with energy cut nHit > 109 (see text for details)

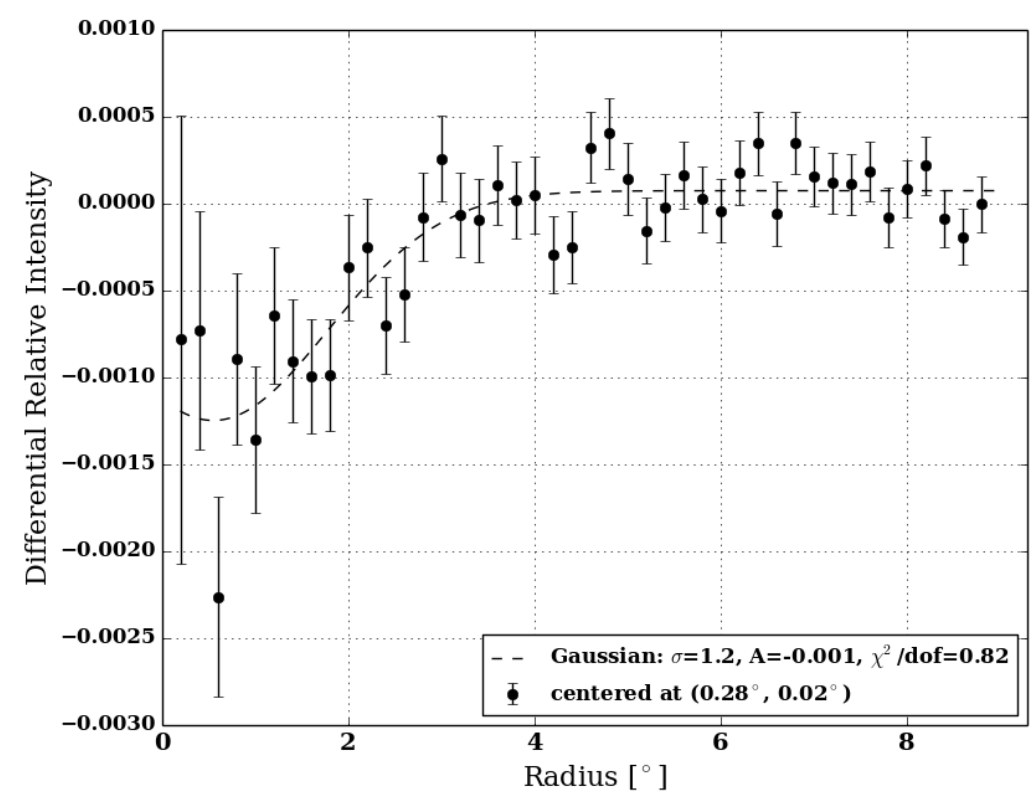

Figure 4: The differential relative intensity of GCRs for energy-proxy bin nHit $>30$, as a function of radius centered at the Sun position. A radial Gaussian fit is adjusted to these points (dashed line). 

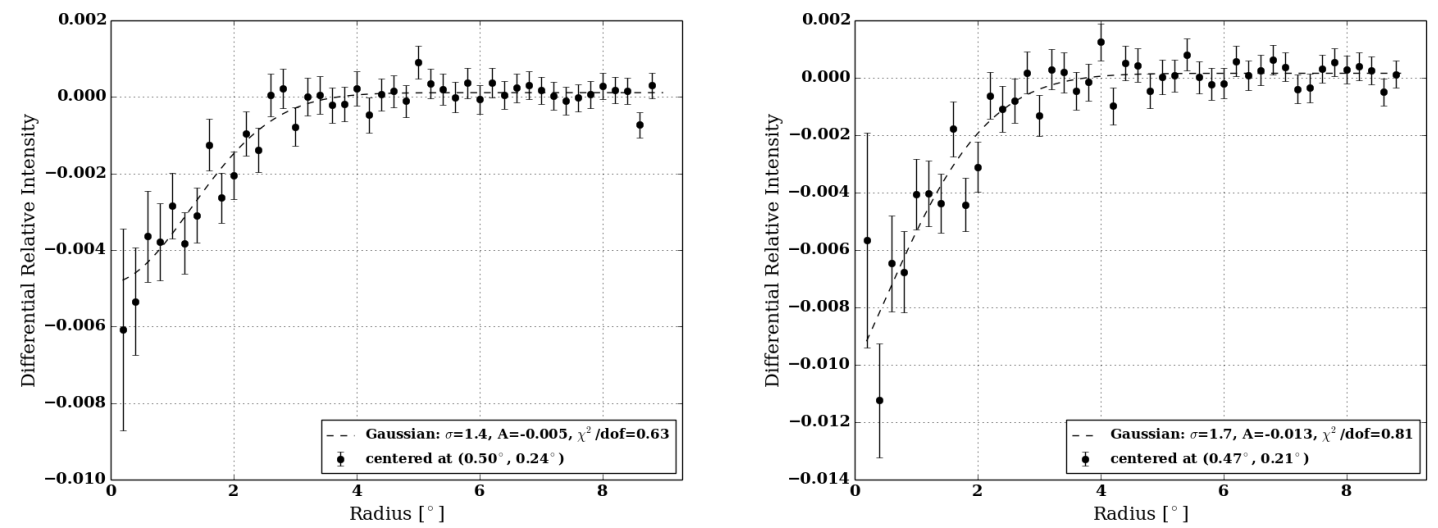

Figure 5: The differential relative intensity of GCRs for energy-proxy bin nHit $>72$ (left panel) and nHit $>109$ (right panel), as a function of radius and centered at the fit Sun position. A radial Gaussian fit to these points is plotted (dashed line).

Table 1: Results of a 2-D asymmetric Gaussian fit of the Sun shadow

\begin{tabular}{crrrrr}
\hline \hline & RA & Dec & & & \\
nHit & $\Delta \alpha$ & $\Delta \delta$ & Width $\Delta \alpha$ & Width $\Delta \delta$ & Amplitude of fit \\
& & & & \\
\hline 30 & $0.28 \pm 0.21$ & $0.023 \pm 0.3$ & $1.30 \pm 0.22$ & $2.18 \pm 0.43$ & $-0.0014 \pm 0.0002$ \\
$>72$ & $0.50 \pm 0.12$ & $0.2412 \pm 0.2$ & $1.10 \pm 0.13$ & $1.59 \pm 0.26$ & $-0.0047 \pm 0.0006$ \\
$>109$ & $0.47 \pm 0.12$ & $0.2184 \pm 0.17$ & $1.08 \pm 0.12$ & $1.52 \pm 0.25$ & $-0.0073 \pm 0.0009$ \\
\hline
\end{tabular}

\section{Summary}

The newly finished HAWC observatory detects both, electromagnetic and hadronic air showers created by cosmic radiation. We have used the hadronic showers to investigate the effects of the Sun and its magnetic field on the cosmic ray flux. Using preliminary data taken during different stages of the construction of the array and which correspond to the maximum phase of the solar cycle 24, we have created maps centered at the Sun position and with a field of view of $20^{\circ}$ in $\Delta \alpha$ and $\Delta \delta$.

We confirmed the existence of a deficit of the GCR flux centered at the Sun. This deficit has been previously observed by other observatories to consist of a strong deficit caused by GCRs directly blocked by the Solar disc (similar in angular size and strength to the Moon shadow) which is weakened by the interaction between GCRs and the Solar magnetic field. This weakening has been correlated with the 11-year Solar cycle.

We plan to use the energy spectrum, time variance (through the solar cycle) and morphology of the Sun shadow maps to obtain information about the coronal magnetic field of the Sun . Measurements of the strength and large-scale morphology of this field are currently unreachable by direct observations at Earth. 


\section{Acknowledgments}

We acknowledge the support from: the US National Science Foundation (NSF); the US Department of Energy Office of High-Energy Physics; the Laboratory Directed Research and Development (LDRD) program of Los Alamos National Laboratory; Consejo Nacional de Ciencia y Tecnología (CONACyT), Mexico (grants 260378, 55155, 105666, 122331, 132197, 167281, 167733); Red de Física de Altas Energías, Mexico; DGAPA-UNAM (grants IG100414-3, IN108713, IN121309, IN115409, IN111315); VIEP-BUAP (grant 161-EXC-2011); the University of Wisconsin Alumni Research Foundation; the Institute of Geophysics, Planetary Physics, and Signatures at Los Alamos National Laboratory; the Luc Binette Foundation UNAM Postdoctoral Fellowship program.

\section{References}

[1] G. W. Clark, Arrival directions of cosmic-ray air showers from the northern sky, Phys. Rev. 108 (Oct, 1957) 450-457.

[2] D. E. Alexandreas, R. C. Allen, D. Berley, S. D. Biller, R. L. Burman, D. R. Cady, C. Y. Chang, B. L. Dingus, G. M. Dion, R. W. Ellsworth, M. K. Gilra, J. A. Goodman, S. Gupta, T. J. Haines, C. M. Hoffman, and et al., Observation of shadowing of ultrahigh-energy cosmic rays by the Moon and the Sun, Physical Review D 43 (Mar., 1991) 1735-1738.

[3] A. Karle, M. Bott-Bodenhausen, A. Kabelschacht, I. Holl, and E. Lorenz, Observation of the Shadowing of Cosmic Rays by the Moon and the Sun, International Cosmic Ray Conference 4 (Aug., 1991) 460.

[4] M. Amenomori et al., Shadowing of Cosmic Rays by the Sun Through the Interplanetary Magnetic Field, International Cosmic Ray Conference 4 (1993) 215.

[5] M. Amenomori et al., A Study of the Shadowing of Galactic Cosmic Rays by the Sun in a Quiet Phase of Solar Activity with the Tibet Air Shower Array, The Asrtrophys. J. 541 (Oct., 2000) 1051-1058, [astro-ph/0008159].

[6] M. Amenomori et al., Variation of Sun shadow in the Solar Cycle 23 observed with the Tibet air shower array, Advances in Space Research 38 (Jan., 2006) 936-941.

[7] M. Amenomori et al., Probe of the Solar Magnetic Field Using the "Cosmic-Ray Shadow" of the Sun, Physical Review Letters 111 (July, 2013) 011101, [arXiv:1306.3009].

[8] F. ZHU, Sun Shadow study in the quiet phase of the solar activity with the ARGO-YBJ experiment, International Cosmic Ray Conference 11 (2011) 158.

[9] X. Xu and MILAGRO Collaboration, The Cosmic Ray Shadows of the Moon and the Sun Detected by the Milagro Gamma Ray Observatory, International Cosmic Ray Conference 7 (July, 2003) 4065.

[10] G. E. Christopher, Physics from the Very-High Energy Cosmic-Ray Shadows of the Moon and Sun with Milagro. PhD thesis, New York University, 2011.

[11] HAWC Collaboration, D. Fiorino, Observation of the Moon Shadow and Characterization of the Point Response of HAWC-30, in Proc. 33th ICRC, (Rio de Janeiro, Brazil), August, 2013.

[12] HAWC Collaboration, A. Abeysekara et al., On the sensitivity of the $\{H A W C\}$ observatory to gamma-ray bursts, Astroparticle Physics 35 (2012), no. 10641 - 650.

[13] K. M. Górski, E. Hivon, A. J. Banday, B. D. Wandelt, F. K. Hansen, M. Reinecke, and M. Bartelmann, HEALPix: A Framework for High-Resolution Discretization and Fast Analysis of Data Distributed on the Sphere, The Asrtrophys. J. 622 (Apr., 2005) 759-771, [a stro-ph/ 0409513 ]. 
[14] D. W. Fiorino, Observation of TeV-Energy Cosmic-Ray Anisotropy with the HAWC Observatory. PhD thesis, The University of Wisconsin - Madison, 2015.

[15] R. Atkins et al., Observation of TeV Gamma Rays from the Crab Nebula with Milagro Using a New Background Rejection Technique, The Asrtrophys. J. 595 (Oct., 2003) 803-811, [astro-ph/0305308].

[16] T.-P. Li and Y.-Q. Ma, Analysis methods for results in gamma-ray astronomy, The Asrtrophys. J. 272 (Sept., 1983) 317-324. 